\title{
Investigation of sources of subnanosecond pulses of the visible spectral range for medical systems
}

\author{
I.V. Zhluktova ${ }^{1}$, V.A. Kamynin ${ }^{1}$, N.R. Arutyunyan ${ }^{1,2}$, A.S. Pozharov ${ }^{1}$, A.I. Trikshev ${ }^{1}$, S.A. Filatova ${ }^{1}$, E.D. \\ Obraztsova $^{1}$, V.B. Tsvetkov ${ }^{1}$ \\ 1. General Physics Institute of Russian Academy of Scinces, ul. Vavilova 38, 119991 Moscow, Russia \\ 2. National Research Nuclear University «MEPHi», Kashirskoe hwy. 31, 115409 Moscow, Russia
}

Currently, fiber laser sources of sub-nanosecond pulses used in many fields of science and technology because of their compactness, efficiency, and manufacturability. And the use of stimulated Raman scattering (SRS) provides the generation outside the spectra of rare-earth elements, making it possible to cover the spectral region from 1 to $2.3 \mu \mathrm{m}$. Thus, in some works, sources with SRS generation both in solid-state [1] and in fiber lasers [2] were demonstrated. This paper presents an investigation hybrid mode-locked of an ytterbium-doped fiber laser (master oscillator) and with generating the 1-st Stokes. The generation of yellow radiation at a wavelength of 530$580 \mathrm{~nm}$ was also obtained using nonlinear crystals from the frequency doubling of the ytterbium-doped laser.

The optical scheme of the experimental setup is shown in Fig.1. The active medium of the MO was pumped using a semiconductor laser diode that emitted at a wavelength of $975 \mathrm{~nm}$. The cavity of the laser was formed by Yb-doped active fiber with a multicomponent cladding (GTWave) and $200 \mathrm{~m}$ single-mode fiber. A pair of polarization controllers were added into the laser cavity to obtain NPE mode-locking. The single-walled carbon nanotubes (SWCNT) were fixed between two optical FC/APC- connectors to realize hybrid mode-locking. The radiation was coupled out from the laser cavity using a polarization beam splitter. As a result, the stable hybrid mode-locking was obtained at a wavelength of $1061 \mathrm{~nm}$. The pulse repetition rate of $1 \mathrm{MHz}$ corresponded to the cavity length. The pulse duration was $200 \mathrm{ps}$. Then, using the $\mathrm{Yb}$-doped fiber amplifier, the average output power at the output of amplifier more than $1 \mathrm{~W}$ was obtained.

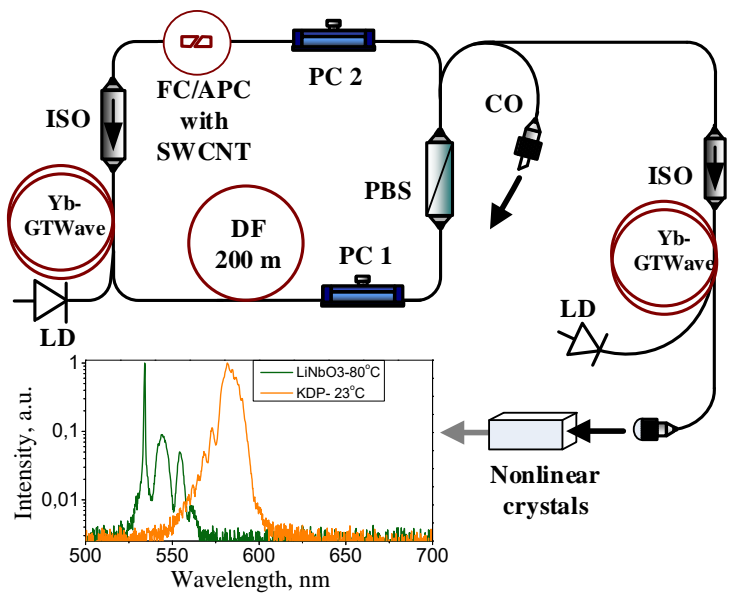

Fig. 1 The optical design of the experimental setup and the spectra obtained by radiation: LD- laser diode, CO- control output, ISO- optical isolator, DF- delay fiber, PC 1,2- polarization controller, PBS- polarized beam splitter.

After amplification of the MO radiation, experiments were performed to the frequency doubling of the ytterbium-doped laser. A KDP(KH2PO4), $\mathrm{CDA}(\mathrm{CsH} 2 \mathrm{AsO} 4)$, and $\mathrm{LiNbO} 3$ crystals were used to get radiation at wavelengths of 530-580 nm. Also, for such crystals as $\mathrm{LiNbO} 3$ and CDA, a heating element with a temperature controller was used to achieve 90-degree synchronism. Also, for such crystals as LiNbO3 and CDA, a heating element with a temperature controller was used to attain 90-degree synchronism because the refractive indices of crystals significantly depend on the temperature of samples. The average output power was up to $1 \mathrm{~mW}$. It can be expected that such a laser may find application in medicine to treat red telangiectasia [3] and in ophthalmology [4].

The reported study was funded by RFBR, project number 19-32-90205.

\section{References}

[1] M. Frank, S. Smetanin, M. Jelínek, D. Vyhlídal, L. Ivleva, P. Zverev, and V. Kubeček, "Highly efficient picosecond all-solid-state Raman laser at 1179 and $1227 \mathrm{~nm}$ on single and combined Raman lines in a BaWO4 crystal, " Optics Lett. 43, 26715-26721 (2018).

[2] W. Liu, P. Ma, H. Lv. J. Xu, P. Zhou, and Z. Jiang, "General analysis of SRS-limited high-power fiber lasers and design strategy, " Opt. Express., 24, 26715-26721 (2016).

[3] N.S. Sadick, and R.Weiss, "The Utilization of a New Yellow Light Laser $(578 \mathrm{~nm})$ for the Treatment of Class I Red Telangiectasia of the Lower Extremities, " Dermatologic Surg., 28 (1), 21-25 (2002).

[4] K. Inagaki, K. Ohkoshi, S. Ohde, G.A. Deshpande, N. Ebihara, A. Murakami, Jpn. J. Ophthalmol. 59, 21 (2015). 